\title{
An Artificial Model for Studying Fluid Dynamics in the Obstructed and Stented Ureter
}

\author{
Carugo D., ElMahdy M., Zhao X., Drake M.J., Zhang X., Clavica F.
}

\begin{abstract}
Fluid dynamics in the obstructed and stented ureter represents a non-trivial subject of investigation since, after stent placement, the urine can flow either through the stent lumen or in the extra-luminal space located between the stent wall and the ureteric inner wall. Fluid dynamic investigations can help understanding the phenomena behind stent failure (e.g. stent occlusions due to bacterial colonization and encrustations), which may cause kidney damage due to the associated high pressures generated in the renal pelvis. In this work a microfluidic-based transparent device (ureter model, UM) has been developed to simulate the fluid dynamic environment in a stented ureter. UM geometry has been designed from measurements on pig ureters. Pressure in the renal pelvis compartment has been measured against three variables: fluid viscosity $(\mu)$, volumetric flow rate $(Q)$ and level of obstruction $(\mathrm{OB} \%)$. The measurements allowed a quantification of the critical combination of $\mu, Q$ and OB\% values which may lead to critical pressure levels in the kidney. Moreover, an example showing the possibility of applying particle image velocimetry (PIV) technology to the developed microfluidic device is provided.
\end{abstract}

\section{INTRODUCTION}

The urinary tract allows for the excretion of urine produced by the kidneys. The ureters are distensible vessels conveying urine from the kidneys to the bladder where the urine is stored until the micturition occurs. The ureteropelvic junction (UPJ) is defined as the junction between the renal pelvis and the ureter while the vesicoureteric junction (VUJ) is defined as the junction between the ureter and the bladder. In healthy conditions the urine is conveyed into the bladder through coordinated ureteric contractions (peristalsis). However, pathological conditions can cause ureteric obstruction such as internal blocks (e.g. stones) or external compressions (e.g. tumours). In these clinical situations,

Carugo D. is with Bioengineering Sciences and Electromechanical Engineering, Faculty of Engineering and the Environment, University of Southampton, Southampton UK (e-mail: D.Carugo@ soton.ac.uk).

ElMahdy M. is with Southmead Hospital, Bristol Urological Institute, Bristol, UK (e-mail: Motaz.Elmahdy@nbt.nhs.uk).

Zhao X. is with Bioengineering Sciences, Faculty of Engineering and the Environment, University of Southampton, Southampton UK; Department of Biomedical Engineering, School of Geosciences and Info-Physics, Central South University, Changsha, China (xf.zhao222@gmail.com).

Drake M. J. is with with University of Bristol, Bristol Urological Institute, Bristol, UK (e-mail: marcus.drake@bui.ac.uk).

Zhang X. is with Bioengineering Sciences, Faculty of Engineering and the Environment, University of Southampton, Southampton UK (e-mail: XL.Zhang@soton.ac.uk).

F. Clavica is with Southmead Hospital, Bristol Urological Institute, Bristol, UK and sector FURORE, Department of Urology, Erasmus MC, Rotterdam, The Netherlands (corresponding author, phone +31 10 7044057; fax +31 10 7044726; e-mail: F.Clavica@erasmusmc.nl). ureteric stents are inserted into the ureter to allow urine drainage from the kidney to the bladder [1-2]. Stents can have short-term applications in patients waiting for spontaneous migration of ureteric stones, or long-term applications in patients with pelvic tumours, inflammatory strictures or retroperitoneal fibrosis [1].

Despite their wide clinical usage, ureteric stents functionality may be severely compromised by the occurrence of complications, including persistent irritation, urinary tract infection due to bacterial colonization on the stent surface (biofilm formation), encrustations as a result of deposition of crystalline urine components [3], stent occlusion [4] and stent migration into the distal ureter [2]. These can have significant impact on the treatment efficacy, patient's life quality and cost of patient care [2]. Double J stents are widely used in the clinical practice, and consist of a hollow tube which traverses the entire ureter and with side holes at regular intervals throughout the whole length.

Understanding the drainage of urine through the stent is non-trivial since, after stent placement, urine can flow either through the stent lumen or in the extra-luminal space located between the stent wall and the ureteric inner wall. Although it has been already postulated that urine flow behaviour may play a critical role in affecting stent functionality [2,4], a full understanding of urine flow dynamics in the obstructed ureter before and after stent positioning has not been fully achieved yet. Few encouraging studies have been conducted in this sense, both numerically [5] and experimentally [6-9]. However, it remains unclear which are the key relevant physical parameters regulating urine flow dynamics in the presence of ureteric stents and how these parameters contribute simultaneously to the progress of stent failure.

In the present study an artificial model of the ureter has been developed, which replicates the physiological fluidic environment in the ureteric tract. The effect of clinicallyrelevant parameters on stent functionality was evaluated via the developed setup. Since abnormally elevated renal pelvic pressures $\left(>20 \mathrm{cmH}_{2} \mathrm{O}\right.$, according to Fung et al. [10]) may contribute to renal damage in the presence of ureteric obstructions, we measured the hydraulic pressure in the renal pelvis model against three variables: (i) fluid viscosity, (ii) level of obstruction, and (iii) fluid flow rate.

\section{METHODOLOGY}

\section{A. Ureter geometry reconstruction}

Ureters were collected from fresh slaughtered domestic pigs. The average age of the animals was 6-8 months. Eight pig ureters were considered to derive average values of lengths and diameters. Each ureter was separated from the 
kidney at the UPJ and from the bladder at the VUJ. The ureters and bladders were collected and stored in Krebs solution at $4^{\circ} \mathrm{C}$ during the transport, before measurements were taken. Each ureter length (L) was divided into 15 equally long segments, and the internal diameters of each segment $\left(\mathrm{D}_{0-15}\right)$ were derived from measurements of the inner perimeter $\left(\mathrm{p}=\pi \mathrm{D}_{\mathrm{i}}\right)$, by opening the ureters with a longitudinal incision (Table I). The average ureter length was equal to $289 \pm 20 \mathrm{~mm}$.

TABLE I:

Average pig ureter internal diameters $\left(\mathrm{D}_{\mathrm{i}}\right.$, in $\left.\mathrm{mm}\right)$, along the longitudinal coordinate $i(i=0$ i.e. $\mathrm{UP} ; i=15$ i.e. $\mathrm{VUJ})$. $\mathrm{N}=8$.

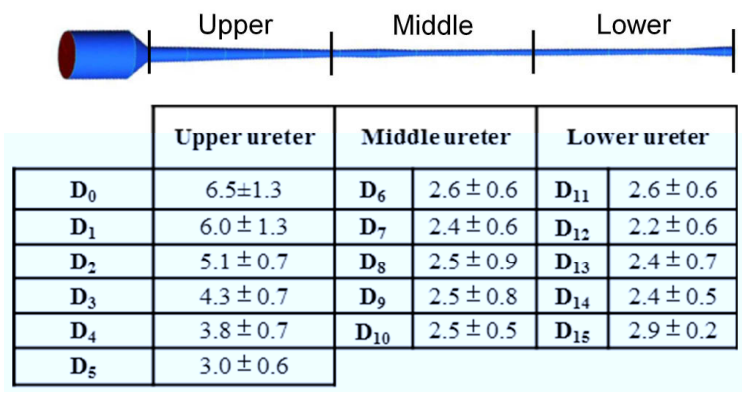

\section{B. Fabrication of the ureter model}

The procedure of ureter model fabrication is described, as follows. Firstly, a Computer-Assisted Design (CAD) of pig ureter was performed using ICEM CFD 12.1 (Ansys Inc., US), based on the average length and diameter values reported in Table I. Furthermore, a cylindrical chamber (diameter: $2.0 \mathrm{~cm}$; height: $3.6 \mathrm{~cm}$ ) reproducing the renal pelvis was designed (kidney side, Fig.1a).

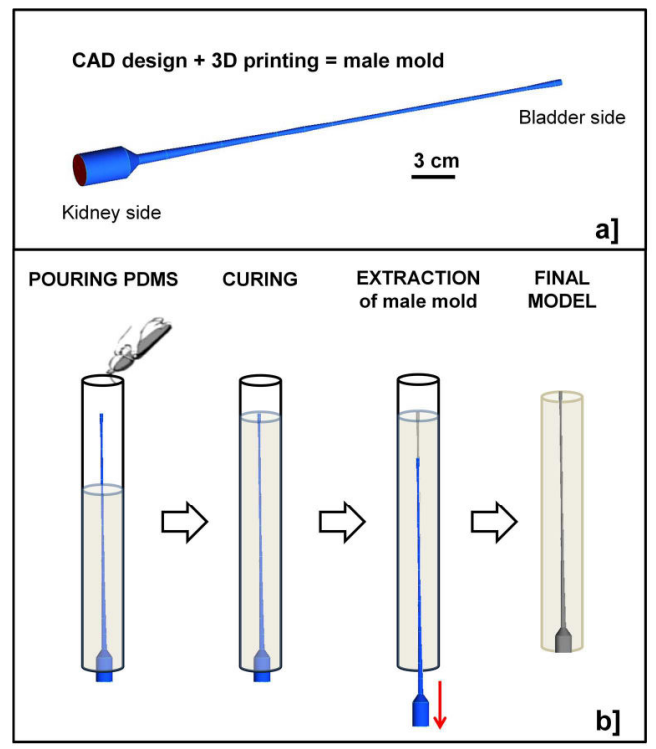

Figure 1 Phases of the fabrication of the PDMS ureteric model (UM).

The CAD geometry was transferred to a 3D printer (Objet Connex $350^{\mathrm{TM}}$, Stratasys Ltd., US) in order to fabricate a rigid male mold of the ureter model. Subsequently, the mold was fixed within a hollow cylinder and a degassed mixture of polydimethylsiloxane (PDMS) precursor and curing agent
(10:1 w/w, Sylgard® 184, Dow Corning Corporation, US) was poured in the cylinder and cured in the oven for $1 \mathrm{~h}$ at $80^{\circ} \mathrm{C}$ [11]. The mold was then removed from the PDMS block and the final model with the imprinted ureter geometry was obtained (Fig.1b). A further curing step of 30min at $100^{\circ} \mathrm{C}$ was performed to achieve complete PDMS curing.

\section{Quantification of pressure in the UM renal pelvis}

A $41 \mathrm{~cm}$ long ureteric stent (Boston Scientific/Microvasive, US) was placed inside the UM with its curling "pigtail" ends located in the renal pelvis and in the bladder compartment, respectively. The stent has an inner diameter of $1.28 \mathrm{~mm}$ and an outer diameter of $2.08 \mathrm{~mm}$. The bladder was modeled as an open end at atmospheric pressure $\left(\mathrm{P}_{\text {bladder }}=0 \mathrm{cmH}_{2} \mathrm{O}\right)$. Measurements of pressure in the renal pelvis compartment of UM were conducted against three different variables: level of obstruction, fluid viscosity and volumetric flow rate. A pressure transducer was employed for this purpose (Gaeltec, UK). The experimental setup is reported in Figure 2.

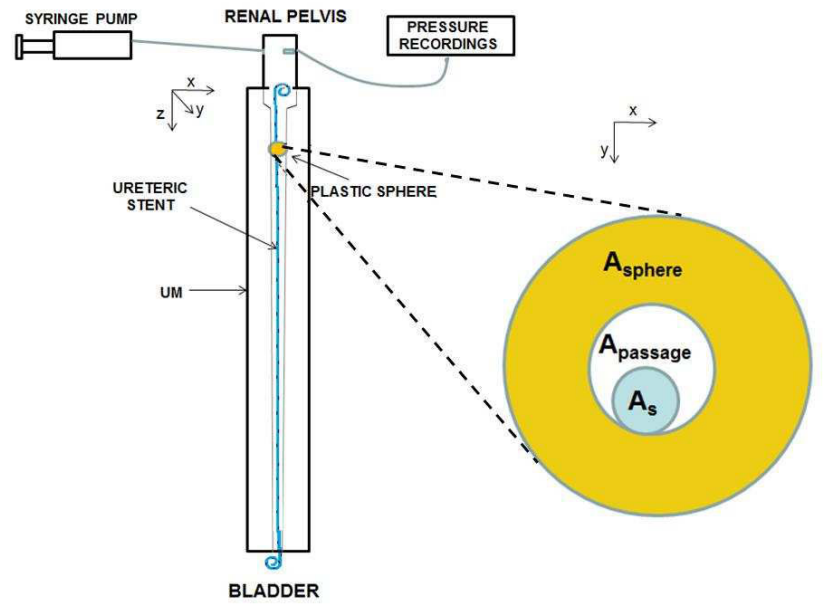

Figure 2 Schematic depiction of the experimental setup and of the plastic sphere employed to simulate a ureteric obstruction.

\section{C.1 Levels of obstruction}

To simulate the fluid dynamics in the obstructed and stented ureter, an occlusion was created in the upper part of the UM by using plastic spheres $\left(\mathrm{D}_{\text {sphere }}=6.07 \pm 0.04 \mathrm{~mm}\right)$. A circular hole was created through the sphere by drilling along the sphere axis, allowing for the stent to pass through the hole. Three different cross sectional areas of the sphere hole ( $\left.\mathrm{A}_{\text {hole }}\right)$ were considered to reproduce different levels of ureteric obstruction. Values of $\mathrm{A}_{\text {hole }}$ and stent cross sectional area $\left(A_{s}=3.4 \mathrm{~mm}^{2}\right)$ were used to calculate the area located between the stent outer surface and the sphere hole inner surface $\left(A_{\text {passage }}=A_{\text {hole }}-A_{s}\right)$ (Fig.2). Considering the sphere cross sectional area $\left(A_{\text {sphere }}=\pi D_{\text {sphere }}{ }^{2} / 4=29.1 \mathrm{~mm}^{2}\right)$, the level of obstruction was calculated as follows (inset, Fig.2):

$$
\text { OB\% }=\left(1-\frac{A_{\text {passage }}}{A_{\text {sphere }}}\right) \cdot 100=\left[1-\frac{A_{\text {hole }}-A_{s}}{A_{\text {sphere }}}\right] \cdot 100
$$

Eq. 1

OB $\%=100 \%$ corresponded to a condition in which $A_{\text {passage }}=0$, with all the fluid passing inside the stent lumen. Three 
different levels of obstruction were considered in the present study, corresponding to $\mathrm{OB} \%=87-90-93 \%$.

\section{C.2 Fluid viscosities and flow rates}

The working fluid corresponded to a solution of glycerol (Sigma Aldrich Co., UK) in deionized water. Five different solutions were produced, each having a different fluid dynamic viscosity (reported in Table II). This allowed to investigate the effect of variations in urine viscosity (e.g. due to bacterial infection or kidney malfunctioning) on renal pelvis pressure in the UM.

\section{TABLE II:}

Dynamic viscosity of the working fluids used in the present investigation, according to the mass proportion of glycerol in water $(\mathrm{G})$ [12]. Values are reported in centipoise $(\mathrm{cP})$; where $1 \mathrm{cP}=0.001 \mathrm{~Pa} \cdot \mathrm{sec} . \mathrm{T}=20^{\circ} \mathrm{C}$.

\begin{tabular}{|c|c|c|c|c|c|c|}
\multicolumn{1}{c|}{} & $0 \% \mathrm{G}$ & $10 \% \mathrm{G}$ & $20 \% \mathrm{G}$ & $30 \% \mathrm{G}$ & $40 \% \mathrm{G}$ & $50 \% \mathrm{G}$ \\
\hline viscosity $(\mathrm{cP})$ & 1 & 1.3 & 1.7 & 2.5 & 3.7 & 6 \\
\hline
\end{tabular}

Four different flow rates $(\mathrm{Q}=5,10,15$ and $20 \mathrm{ml} / \mathrm{min})$ were enforced using a syringe pump (KD Scientific, UK), for each combination of the specified fluid viscosities and obstruction levels. Ureteral flow rate values for pigs were chosen within the range $0-20 \mathrm{ml} / \mathrm{min}$, coherently with Tofft et al. [13].

\section{Flow visualization experiments}

The UM was placed on the stage of a fluorescent microscope (Olympus Corporation, Japan) and microscope focus was set on channel mid plane. Fluorescent polystyrene beads $(20 \mu \mathrm{m}$ diameter; Polysciences, Inc., US) were added to the working fluid. Beads were exposed to fluorescent light (excitation wavelength, $\lambda_{e x}=441 \mathrm{~nm}$ ) and emitted light with a wavelength, $\lambda_{e m}$, of $486 \mathrm{~nm}$. By using an optical filter, only the fluorescent images of beads were acquired by an high speed CCD camera (Pixelfly, PCO AG, Germany), with a spatial resolution of $1392 \times 1024$ pixels $\times$ pixels. A $10 x$ magnification objective was employed for this purpose. Images were acquired in close proximity to a side hole of the stent, positioned just after the obstruction.

\section{RESULTS}

Surface plots of Figure $3 a$ and $3 b$ show the combined effect of fluid dynamic viscosity (in $\mathrm{cP}$ ) and volumetric flow rate (in $\mathrm{ml} / \mathrm{min}$ ) on the hydraulic pressure in the renal pelvis of UM, at two different levels of model obstruction, namely OB $\%=87$ (Fig. 3a) and OB\%=93 (Fig. 3b). Values have been derived from linear interpolation of the experimental data points acquired at the experimental conditions reported previously. Iso-pressure lines at 20,40 and $60 \mathrm{cmH}_{2} \mathrm{O}$ are also reported on the graph. Figure 4 shows the effect on renal pelvis pressure caused by an increasing level of obstruction and fluid viscosity. Three different levels of obstruction have been considered, corresponding to $\mathrm{OB} \%=87,90$ and 93 . Figure 5 instead shows a representative microscope image of $20 \mu \mathrm{m}$ diameter fluorescent polystyrene beads (Polysciences Inc., US) flowing within the UM.

\section{DISCUSSION}

Only few studies have attempted to dynamically model ureteric stents exposed to flow in vitro [14]. In this work a biomimetic physical model, closely resembling the real ureter geometry, has been designed based on direct measurements on pig ureters. It therefore represents a first attempt to simulate experimentally the fluid dynamics in the obstructed and stented ureter. The choice of designing UM architecture based on pig ureter geometry - rather than human ureter - represents a starting point which will allow us to potentially validate the UM by performing analogous experiments in-vitro using easily-accessible pig ureters. After validation, the developed physical model may be of interest to clinicians to understand and quantify the role of individual physical variables on the renal pressure, under a range of clinically relevant conditions (i.e. unobstructed, obstructed or stented ureter).
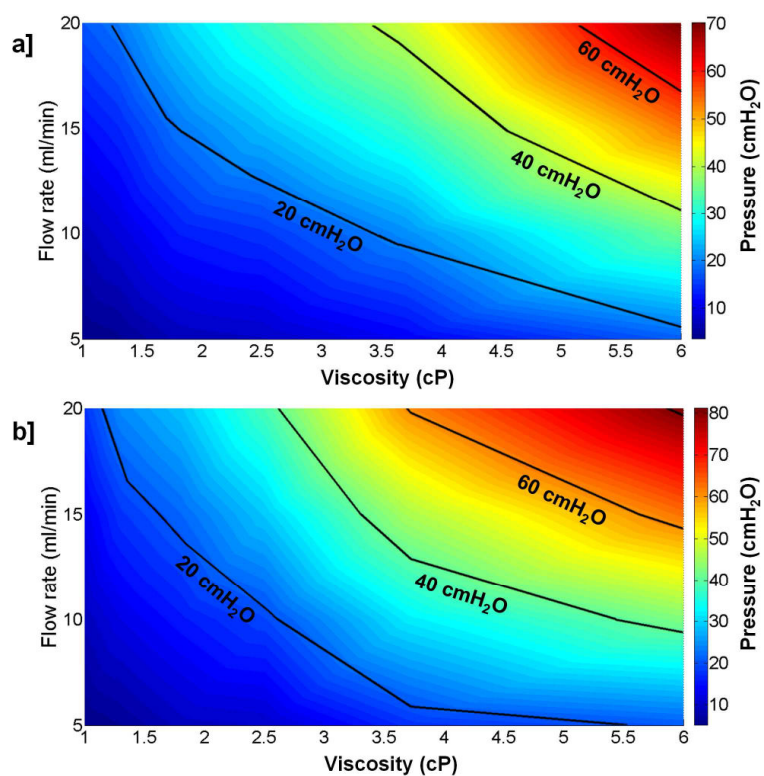

Figure 3 Colourmap illustrating the dependence of the hydraulic pressure in the renal pelvis of UM, on both the fluid viscosity (in $\mathrm{cP}$, x-axis) and the fluid flow rate (in $\mathrm{ml} / \mathrm{min}, \mathrm{y}$-axis). Two levels of obstruction have been considered, corresponding to $\mathrm{OB} \%=87$ (a) and $\mathrm{OB} \%=93$ (b). Colours correspond to different pressure values, reported in the colourbar on the right hand side. Iso-pressure lines at 20,40 and $60 \mathrm{cmH}_{2} \mathrm{O}$ are reported (black lines). $\mathrm{N}=3$.

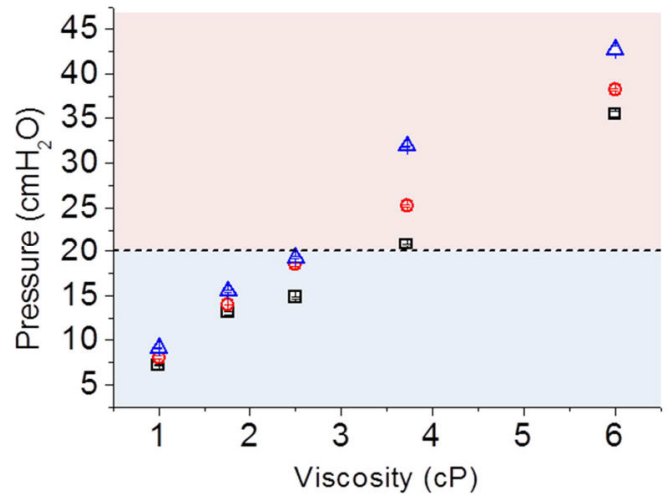

Figure 4 Dependence of the hydraulic pressure in the UM renal pelvis on the fluid dynamic viscosity, at three different levels of obstruction, corresponding to $\mathrm{OB} \%=87$ (black squares), 90 (red circles) and 93 (blue triangles). The fluid flow rate was set to an average value of $10 \mathrm{ml} / \mathrm{min}$. The black dotted line corresponds to a fixed critical pressure of $20 \mathrm{cmH}_{2} \mathrm{O}$. The pink area above the line corresponds to potential kidney damage, whilst the blue area below the line corresponds to physiological kidney function. $\mathrm{N}=3$. 
Figure 3, for example, allows a clear identification of the combined values of fluid viscosity and flow rate which result in a safe/dangerous pressure for the kidney. Importantly, this analysis can be performed at different levels of ureteric obstruction. This can be verified by identification of the combined values of $\mu$ and $Q$ for which the pressure is located below or above the iso-pressure line at $20 \mathrm{cmH}_{2} \mathrm{O}$ [10], corresponding to correct kidney functioning ("safe area") or potential kidney damage, respectively. Moreover, the comparison between Figure $3 \mathrm{a}$ and Figure $3 \mathrm{~b}$ clearly illustrates how a small increase in the level of obstruction (e.g. from $87 \%$ to $93 \%$ ) significantly affected the size of the "safe area" with a more limited range of variations, for both $\mu$ and Q, in the case of higher obstruction levels. Notably, it can be observed as for both levels of obstruction and viscosity = $1 \mathrm{cP}$ (close to the normal urine viscosity), the pressure in the renal pelvis is always inside the "safe area" for any combination of $\mathrm{Q}$ and $\mu$, suggesting that the ureteric stent is performing effectively at the lower fluid viscosities. However, with increasing the fluid viscosity, the pressure in the UM renal pelvis increased almost linearly, as illustrated in Figure 4. Specifically, at an average $\mathrm{Q}=10 \mathrm{ml} / \mathrm{min}$, the pressure overcame the critical value of $20 \mathrm{cmH}_{2} \mathrm{O}$ at $\mu>3 \mathrm{cP}$, suggesting that in the case of pathological increase of urine viscosity the ureteral stent may not be capable of maintaining the pressure in the renal pelvis below potentially critical values for correct kidney functioning.

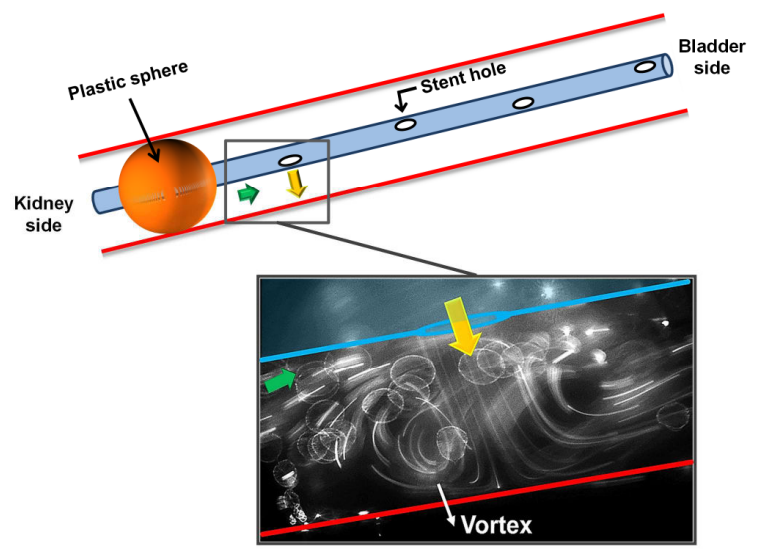

Figure 5 Representative microscope image of $20 \mu \mathrm{m}$ diameter fluorescent beads flowing within the UM in close proximity to a side hole of the stent, located after the UM obstruction (at $\mathrm{OB} \%=97$ ). The fluid flow rate was set to $5 \mathrm{ml} / \mathrm{min}$. The blue line and the red line correspond to the stent wall and the UM inner wall, respectively. The direction of fluid flowing from the intraluminal to the extra-luminal region of the stent is indicated by the yellow arrow. The green arrow indicates the presence of residual fluid flow in the extra-luminal space, due to incomplete obstruction.

Further analyses will include the evaluation of fluid flow fields within the UM, by using high-resolution imaging techniques. With this respect, the representative microscope image reported in Figure 5 illustrates the fluid flow characteristics in a region located nearby a stent side hole, located just after UM occlusion. It is possible to observe both the presence of residual fluid flow in the extra-luminal space (green arrow) and of fluid flowing from the intraluminal to the extra-luminal space of the stent (yellow arrow). Interestingly, the formation of vortices in the extraluminal space has been detected, which can potentially influence the dynamics of crystals and biofilm deposition on the stent surface. This will represent the subject of future investigations.

\section{LIMITATIONS}

Although we acknowledge the distensibility of the ureter, in this work we employed a rigid material to fabricate the UM, due to the large number of variables involved. Further improvements to the UM may include an appropriate dosing of PDMS precursor and curing agent to match the natural distensibility of the ureter. Although in this work the ureteric peristalsis has been neglected, we consider that the modelled conditions can still resemble the fluid dynamic of a stented ureter since it has been observed that stent insertion results in a pronounced reduction of peristalsis (particularly in the long-term) $[1,5]$.

\section{REFERENCES}

[1] A.-C. Kinn and H. Lykkeskov-Andersen, "Impact on ureteral peristalsis in a stented ureter. An experimental study in the pig", Urol Res, vol. 30, pp. 213-218, 2002.

[2] G. Haleblian, K. Kijvikai, J. de la Rosette and G. Preminger, "Ureteral stenting and urinary stone management: A Systematic Review", The Journal of Urology, vol. 179, pp. 424-430, 2008.

[3] K.A. Hausegger and H.R. Portugaller, "Percutaneous nephtostomy and antegrade ureteral stenting: technique - indications - complications", Eur Radiol., vol. 16, pp. 2016-2030, 2006.

[4] S.G. Docimo and W.C. Dewolf, "High failure rate of indwelling ureteral stents in patients with extrinsic obstruction: experience at 2 institutions", The Journal of Urology, vol. 142, pp. 277-279, 1989.

[5] S.L. Waters, K. Heaton, J.H. Siggers et al., "Ureteric stents: investigating flow and encrustation", J. Engineering in Medicine, vol. 222, pp. 551-561, 2008.

[6] J.W.A. Ramsay, S.R. Payne, P.T. Gosling et al., "The Effects of Double J stenting on unobstructed ureters", British Journal of Urology, vol. 57, pp. 630-634, 1985.

[7] H.K. Mardis, R.M. Kroeger, T.W. Hepperlen, et al., "Polyethylene double pigtail ureteral stents", Urologic Clinics of North America, vol. 9, pp. 95-101, 1982.

[8] G. Watson, "Problems with Double-J Stents and Nephrostomy Tubes", Journal of Endourology, vol. 11, pp. 413-417, 1997.

[9] H. Fine, R.L. Gordon and P.D. Lebensart, "Extracorporeal shock wave lithotripsy and stents: fluoroscopic observations and a hypothesis on the mechanisms of stent function", Urol Radiol., vol. 1, 37-41, 1989.

[10] L.C. Fung, A. Atala, "Constant elevation in renal pelvic pressure induces an increase in urinary N-Acetyl-Beta-D-Glucosaminidase in a nonobstructive porcine model", J. Urol, vol. 159(1), pp. 212-216, 1998.

[11] X. Zhang and S.J. Haswell "Materials matter in microfluidic devices", MRS Bulletin, vol. 31(2), pp. 95-99, 2006.

[12] J.B. Secur and H.E. Oberstak, "Viscosity of glycerol and its acqueos solutions", Industrial and Engineering Chemistry, vol. 43, pp. 2117$2120,1951$.

[13] H.P. Tofft, J. Frokier, J. Mortensen and J.C. Djurhuus, "Renal pelvic peristalsis in pigs during standardized flow rate variations", Urol. Int., vol. 41, pp. 292-298, 1986.

[14] S.K.S. Choong, S. Wood and H.N. Whitfield, "A model to quantify encrustation on ureteric stents, urethral catheters and polymers intended for urological use", BJU Intern., vol. 86, pp. 414-421, 2000. 